\title{
COVID-19: consideraciones en el manejo de la vía aérea en pacientes pediátricos
}

\section{Covid-19: considerations in the management of airway in pediatric patients}

Eloy Sánchez-Hernández, ${ }^{1}$ Regina Rodríguez-Arias, ${ }^{2}$ Karla M Lara-Cuevas, ${ }^{3}$ Néstor A. Camacho -Jaramillo ${ }^{3}$

\section{Resumen}

Revisión de la bibliografía del coronavirus (COVID-19) y su relación con el paciente pediátrico ante la pandemia. Este material, y su aplicación en niños, tiene como propósito ofrecer orientación a los profesionales de nuestro hospital y sugerencias para tratar a los niños que requieran atención, bien sea solo para intubación por complicaciones respiratorias o, bien, porque requieren un procedimiento quirúrgico para resolver algún problema no relacionado con la crisis sanitaria actual.

PALABRAS CLAVE: Coronavirus; COVID-19; paciente pediátrico; pandemia; guía para profesionales; intubación.

\section{Abstract}

The present work is the result of the review of publications on the coronavirus, known as COVID-19, and its role in the paediatric patient in the face of the pandemic that the world is currently experiencing.

The present material and its application in children, aims to provide guidance to professionals in our hospital, as well as suggestions for approach and management of children who require attention, either only for intubation for respiratory complications or because they will undergo a surgical procedure for some problem not related to the health crisis that concerns us today.

KEYWORDS: Coronavirus; COVID-19; Paediatric Patient; Pandemic; Guidance to Professionals; Intubation

\author{
${ }^{1}$ Anestesiólogo Pediatra, Maestría en \\ Educación Basada en Competencias \\ en la Universidad del Valle de México \\ (título en trámite), Hospital Civil de \\ Guadalajara "Fray Antonio Alcalde". \\ Guadalajara, Jalisco. \\ ${ }^{2}$ Neuroanestesióloga, Maestría en \\ Investigación clínica en la Universi- \\ dad de Guadalajara, Hospital Civil de \\ Guadalajara "Fray Antonio Alcalde". \\ Guadalajara, Jalisco \\ ${ }^{3}$ Médico residente del 3er. año de la \\ especialidad de Anestesiología, Hospi- \\ tal Civil de Guadalajara "Fray Antonio \\ Alcalde". Guadalajara, Jalisco.
}

Recibido: 14 de mayo de 2020

Aceptado: 05 de junio de 2020

Correspondencia

Eloy Sánchez-Hernández dreloy124@hotmail.com

Este artículo debe citarse como Sánchez-Hernández E, RodríguezArias R, Lara-Cuevas KM, Camacho -Jaramillo NA. COVID-19: consideraciones en el manejo de la vía aérea en pacientes pediátricos. Acta Pediatr Méx 2020; 41 (Supl 1):S72-S80.

\section{ANTECEDENTES}

Hay que tener en mente que la cantidad de pacientes pediátricos que en el mundo han requerido hospitalizarse debido al COVID-19 es muy baja, ${ }^{1}$ pero esto no debe resultar limitativo porque la posibilidad está latente.

Las sugerencias para su atención se basan en las características de nuestro hospital y pueden, o no, aplicarse en otros entornos hospitalarios. Al igual que mucha de la información disponible hoy en día, está sujeta a cambios y adecuaciones dependientes de las publicaciones que, día a día, se actualizan, así como del criterio del equipo médico que atenderá el caso.

La información disponible para población pediátrica es mínima, no solo en relación con el comportamiento de la enfermedad, sino también de la manera en que debe tratarse; aún no se ha acumulado la suficiente experiencia necesaria 
para emitir recomendaciones; hoy solo es posible externar sugerencias.

El coronavirus es la enfermedad que tiene como fuente más importante de trasmisión el medio aéreo, a través de partículas flotantes (aerosoles), por contacto directo con pacientes o superficies (vectores pasivos) contaminadas ${ }^{2}$ y que afecta a toda la población, pero con mayor letalidad a la adulta, diferente a lo que ocurre en la pediátrica, en la que los síntomas son leves (incidencia en niños de $0.9 \%)^{3,4}$

El nivel de bioseguridad catalogado para la pandemia es nivel III (nivel alto de contagiosidad), que implica trasmisión por vía respiratoria (aerosol) y uso obligatorio de equipo de protección personal para tratar a estos pacientes. ${ }^{5}$

Al ser la vía de contagio por medio de los aerosoles generados durante la manipulación de la vía aérea y que, en el entorno hospitalario del niño, ésta es ampliamente utilizada, no solo para intubación sino también durante el tratamiento de diferentes enfermedades que requieren medidas terapéuticas y que no es raro que se estén nebulizando o que reciban soporte ventilatorio, bien sea de forma no invasiva o en los casos más delicados con ventilación con altos flujos por vía nasal. Además de lo anterior están los procedimientos altamente generadores de aerosoles: broncoscopias, endoscopias digestivas, cirugía laparoscópica, uso de cauterio o la simple aspiración de secreciones de las vías respiratorias.

\section{Objetivos}

Ofrecer una propuesta de atención con la finalidad de llegar a un consenso para el tratamiento de niños en nuestro hospital que requieran manipulación de la vía aérea en urgencias, área COVID o en el quirófano.

\section{Alcance}

Todos los pacientes pediátricos con infección por COVID-19 confirmada o sospechada, que requieran manipulación de la vía aérea debido a su gravedad o por requerir algún procedimiento quirúrgico, médico o de imagen que haga necesaria la anestesia general con manejo de la vía aérea en el Antiguo Hospital Civil Fray Antonio Alcalde.

\section{Equipo protección personal}

La adecuación del equipo de protección se ha convertido en una práctica obligatoria y evolutiva porque habitualmente, cuando se atendía a pacientes con riesgo de contaminación por aerosoles, era limitada (bata no impermeable, guantes, protectores oculares y cubrebocas N95). Está comprobado que no es suficiente, por eso se incrementó el equipo necesario que abarcara a cubrir la cabeza y el cuello; se agregó doble guante, cubierta de cabeza y cuello con capucha quirúrgica (escafandra), bata de protección grado 3 (impermeable) y cubierta de calzado. ${ }^{6}$

Por lo que se refiere al equipo de protección personal se han publicado varias guías, todas para pacientes adultos pero que también pueden aplicarse a los enfermos pediátricos. ${ }^{7}$

Después de revisar varias guías referentes a los criterios de vestimenta y sugerencia de secuencia de instalación y retiro del equipo de protección personal, nos apegamos a ellas. Anexo 1 y $\mathbf{2}$

\section{Manejo de la vía aérea}

La manipulación de la vía aérea en niños, por sí sola, puede implicar todo un reto para el anestesiólogo o especialista porque las características anatómicas propias de la edad son cambiantes. Por eso las maniobras debe ejecutarlas el espe- 


\section{Colocación del equipo de protección personal}

Antes de iniciar, los operadores de vía aérea deberán retirarse artículos personales (anillos, pulseras, aretes, reloj, etc.). Hombres sin barba ni bigotes.

El verificador leerá de forma lenta y sistemática los pasos

1. Realizar higiene de manos. Lavado de manos con solución sanitizante por 30 segundos.

2. Colocar mascarillas N95. Realizar prueba de fuga.

3. Calzar primer par de guantes

4. Calzar botas de protección internas. Asegurarse que cubran por completo el calzado y la parte interna del pantalón.

5. Calzar gorro impermeable. Asegurarse de cubrir cabello.

6. Calzar traje de aislamiento. Subir cremallera sin calzar la capucha

7. Calzar botas de protección externas. Cubrir la parte inferior del traje de protección.

8. Calzar segundo par de guantes. Cuidar y cubrir mangas del equipo de producción.

9. Colocar lentes de protección. Deben sellar perfectamente alrededor de los ojos del operador.

10. Colocar mascarilla quirúrgicas. Sobre N95

11. Calzar capucha del traje de aislamiento. Asegurarse de cubrir las orejas

12. Colocar bata de protección impermeable o mandil. Ajustar parte superior, anudar cintas de manera lateral, cubrir espalda y asegurar segundo par de guantes con manga de la bata.

13. Calzar tercer par de guantes

14. Colocar careta de protección

Los operadores llevarán a cabo una inspección del equipo con ayuda del verificador, asegurándose de no dejar zonas del cuerpo descubiertas, realizando arcos de moviemiento de cabeza, cuello y extremidades superiores.

Anexo 1. Colocación del equipo de protección personal.

cialista con mayor experiencia y adiestramiento en el trato con niños. Es igualmente recomendable que el segundo operador o ayudante también cuente con la misma preparación y ambos estén familiarizados con el equipo y procedimiento correcto de protección personal.

Los dispositivos de aislamiento para disminuir la liberación de aerosoles durante la intubación también son aplicables a este grupo poblacional para conseguir mejor control con el campo de aislamiento transparente.

\section{Inducción}

Se apega a la directriz de inducción, con secuencia rápida para la intubación, con la finalidad de disminuir la generación de aerosoles durante 


\section{Retiro del equipo de protección personal}

El equipo se retira del tal forma que las áreas expuestas queden hacia el interior y evitar el contacto con las mismas, todas se dispondrán en bolsa para desechar

El verificador leerá de forma lenta y sistemática los pasos

1. Sanitización de manos (30 segundos)

2. Retiro de careta. Sanitización de manos (30 segundos)

3. Retiro de tercer par de guantes. Sanitización de manos (30 segundos)

4. Retiro de bata de protección. Sanitización de manos (30 segundos)

5. Retiro de capucha de equipo de producción. Sanitización de manos (30 segundos)

6. Retiro de mascarilla quirúrgica. Sanitización de manos (30 segundos)

7. Retiro de lentes de producción. Sanitización de manos (30 segundos)

8. Retiro de botas. Sanitización de manos (30 segundos)

9. Bajar cierre de traje de protección blanco. Sanitización de manos (30 segundos)

10. Retiro de segundo par de guantes. Sanitización de manos (30 segundos)

11. Retiro de traje de traje de protección. Sanitización de manos (30 segundos)

12. Retiro de gorro asegurando de no manipular la parte externa. Disponer del mismo de inmediato

13. Retiro de botas internas. Sanitización de manos (30 segundos)

14. Retiro de guantes internos. Sanitización de manos (30 segundos)

Anexo 2. Retiro del equipo de protección personal.

el procedimiento; lo importante es pre-oxigenar sin dar ventilación positiva con la mascarilla. La ventilación positiva se inicia cuando el paciente ya está intubado, con sellado de la vía aérea con balón de cánula endotraqueal.

Los fármacos de inducción deben administrarse a la dosis que corresponda al paciente, conforme a su peso actual; es importante pesarlo o estimar su peso. El segundo operador debe estar familiarizado con los procedimientos, medicamentos y dosis que deben administrarse. Cuadro 1
Cuadro 1. Dosis de los fármacos inductores

\begin{tabular}{l|c|c|}
\hline \multicolumn{3}{|c|}{ Inductor } \\
\hline Propofol & Ketamina & Etomidato \\
\hline $2-3 \mathrm{mg} / \mathrm{kg} / \mathrm{IV}$ & $1-2 \mathrm{mg} / \mathrm{kg} / \mathrm{IV}$ & $0.2-0.3 \mathrm{mg} / \mathrm{kg} / \mathrm{IV}$ \\
\multicolumn{3}{|c|}{ Bloqueador neuromuscular } \\
Rocuronio & Vecuronio & Succinilcolina \\
$0.6-1$ & $0.1-0.15$ & $1-1.5 \mathrm{mg} / \mathrm{kg} / \mathrm{IV}$ \\
$\mathrm{mg} / \mathrm{kg} / \mathrm{IV}$ & $\mathrm{mg} / \mathrm{kg} / \mathrm{IV}$ \\
& Opioide \\
fentanil & \\
$2-3 \mathrm{mcg} / \mathrm{kg}$ & $0.1-0.3 \mathrm{mcg} / \mathrm{kg} / \mathrm{IV}$
\end{tabular}




\section{Intubación}

El tamaño de la cánula traqueal debe elegirse de forma correcta. Si bien existen múltiples tablas de relación edad-tamaño de cánula, y diferentes fórmulas, aquí se proporciona una de cálculo rápido y una tabla de relación que permite obtener el tamaño adecuado de la cánula a colocar. Esto puede enriquecerse si se usa la hoja o pala de laringoscopio adecuado, conforme a la edad del paciente.

Es importante tener en mente que todas las cánulas deben tener globo. Debe medirse la presión de sello del globo para no exceder los $20 \mathrm{~cm}$ de agua. Cuadro 2 y 3

Calculo cánula endotraqueal:

$\frac{\text { Edad }}{4}+3.5=$ número de cánula con globo

Es necesario tener presente la posibilidad de no poder intubar o de un efecto adverso, como el laringoespasmo, que es frecuente en niños, con

Cuadro 2. Tamaño de cánula según edad

\begin{tabular}{|l|c|}
\hline \multicolumn{2}{|c|}{ Cánula } \\
\hline Tamaño & Edad Recomendada \\
\hline $3.0 \mathrm{~mm}$ & $\begin{array}{c}\text { Menores de } 3 \mathrm{~kg} \text { y menores } \\
\text { de } 8 \text { meses }\end{array}$ \\
\hline $3.5 \mathrm{~mm}$ & De 8 meses a 2 años \\
\hline $4.0 \mathrm{~mm}$ & $2-4$ años \\
\hline $4.5 \mathrm{~mm}$ & $4-6$ años \\
\hline
\end{tabular}

Cuadro 3. Dispositivos auxiliares para intubación

\begin{tabular}{lc}
\hline \multicolumn{2}{c}{ Otros dispositivos } \\
\begin{tabular}{lc}
\hline Cánula de Guedel & $00,0,1,2,3$ y 4 \\
\hline Guía para cánula endotraqueal & 6 French \\
(tubo traqueal 2.5 a 4.5$)$ \\
10 French \\
(tubo traqueal 5 más)
\end{tabular} \\
\end{tabular}

incidencia global de $0.87 \%$. Es mayor $(2.82 \%)$ en pacientes menores de 3 meses (intubación esofágica, bradicardia secundaria a hipoxia). En estas circunstancias puede considerarse el uso de mascarilla laríngea del tamaño adecuado al paciente. El dispositivo supraglótico no garantiza el sellado de la vía aérea. ${ }^{8}$ Cuando no sea posible intubar debido a un espasmo laríngeo podrá recurrirse a la ventilación asistida con mascarilla facial, con la posibilidad de generar aerosoles. Por esto es necesario estar seguros de continuar con el equipo de protección completa, incluida la cubierta plástica para contención de aerosoles. Cuadros 4 y 5

\section{Oxigenación}

La oxigenación del paciente pediátrico durante el traslado a la sala de quirófano, o previa a la

Cuadro 4. Tamaño de hoja de laringoscopio según edad

\begin{tabular}{|l|c|c|}
\hline \multicolumn{3}{|c|}{ Hoja de laringoscopio para intubación } \\
\hline Edad & Miller (recta) & Macintosh (curva) \\
\hline Prematuro & 00 & ----- \\
\hline Neonato & 0 & ----- \\
\hline Neonato a 2 años & 1 & ---- \\
\hline $2-6$ años & 2 & ---- \\
\hline $6-12$ años & $2-3$ & 2 \\
\hline Mas de 12 años & 3 & 3 \\
\hline
\end{tabular}

Cuadro 5. Tamaño de la mascarilla laríngea según el peso

\begin{tabular}{|l|c|c|}
\hline \multicolumn{3}{|c|}{ Mascarilla laringea } \\
\hline Tamaño & $\begin{array}{c}\text { Peso del paciente } \\
\text { en kg. }\end{array}$ & $\begin{array}{c}\text { Volumen máximo de } \\
\text { aire en balon }\end{array}$ \\
\hline $\mathbf{1}$ & Menos de $5 \mathrm{~kg}$ & $4 \mathrm{ml}$ \\
\hline $\mathbf{1 . 5}$ & 5 a 10 & $7 \mathrm{ml}$ \\
\hline $\mathbf{2}$ & 10 a 20 & $10 \mathrm{~mL}$ \\
\hline $\mathbf{2 . 5}$ & $20-30$ & $14 \mathrm{~mL}$ \\
\hline $\mathbf{3}$ & $30-50$ & $20 \mathrm{~mL}$ \\
\hline $\mathbf{4}$ & $50-70$ & $30 \mathrm{~mL}$ \\
\hline $\mathbf{5}$ & $70-100$ & $40 \mathrm{~mL}$ \\
$\mathbf{6}$ & $>100$ & $50 \mathrm{~mL}$
\end{tabular}


intubación en su cubículo de hospitalización o sala de choque, es de gran relevancia (recuérdese la pobre tolerancia a la hipoxia en este grupo poblacional) y debe tenerse en mente el escenario en que se estará atendiendo al paciente.

\section{En cubículo de hospitalización o sala de choque.}

La aplicación de estrategias de ventilación no invasiva a niños ha sido de gran utilidad porque se evita intubarlos en escenarios no relacionados con el coronavirus. Como parte de la ventilación no invasiva está la "ventilación de alto flujo por vía nasal". Esta vía de suministro de oxígeno es un procedimiento que genera aerosoles; por eso no se recomienda iniciarlo como parte del proceso de intubación. Si el paciente ya lo tiene instalado puede dejarse y continuar su administración ${ }^{1}$ porque si se suprime puede agravarse su condición. Si el escenario es otro, en el que el paciente esté recibiendo soporte de oxígeno a través de otro dispositivo y esté sin intubar o será por traslado al quirófano, se recomienda el soporte de oxígeno con flujos bajos (menos de $5 \mathrm{~L} / \mathrm{min}$ ) porque esta práctica es poco probable que genere aerosoles; esto obliga a extremar precauciones a todo el personal que participa en la intervención.

Traslado al quirófano. Es recomendable hacerlo con soporte de oxígeno a bajo flujo, por medio de puntas nasales y cubrebocas (Figura 1) o, en su defecto, en pacientes pequeños el uso de casco cefálico (escafandra) con soporte de oxígeno y cubrir la parte del cuello con un campo que haga las veces de sistema parcialmente cerrado (Figura 2) que contenga los aerosoles que pudieran estarse generando.

Se recomienda que el traslado sea directo del cubículo al quirófano (de preferencia no pasar por recuperación o sala de preparación prequirúrgica). ${ }^{7}$

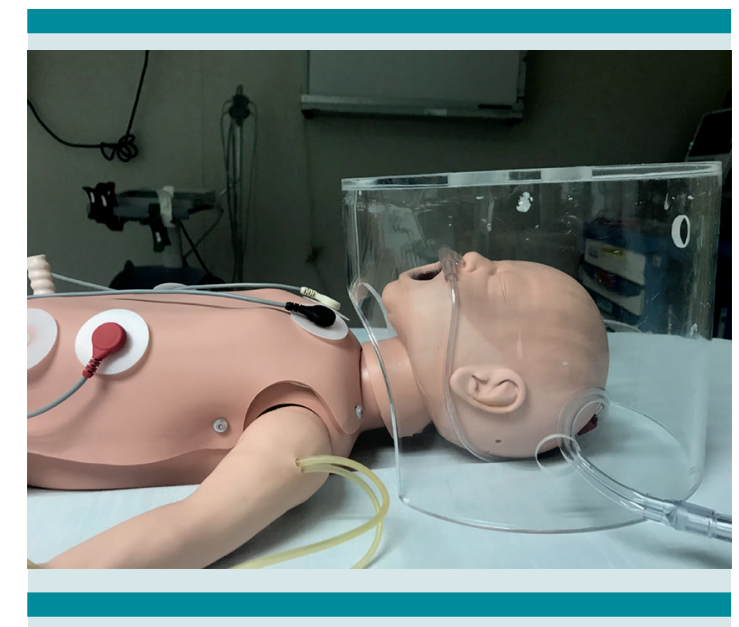

Figura 1. Puntas nasales de alto flujo y escafandra para contención de aerosoles.

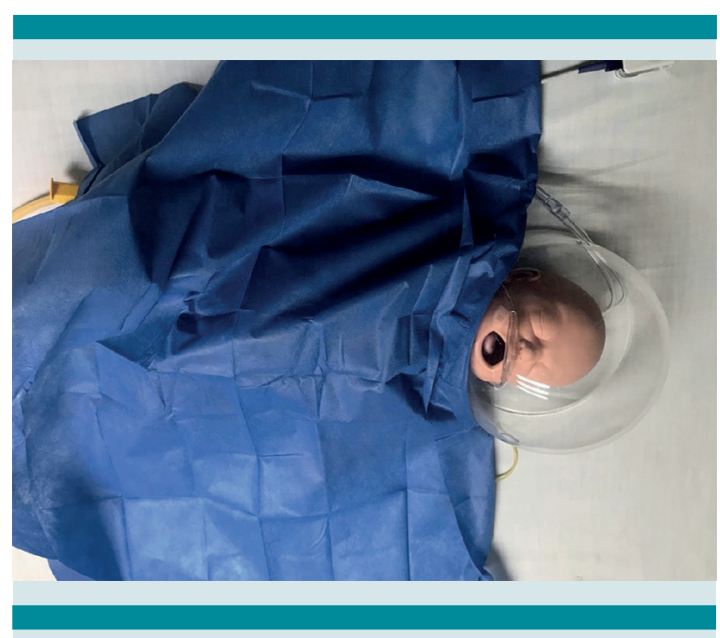

Figura 2. Puntas nasales de alto flujo, escafandra y cubierta aislante para evitar aerosoles durante el traslado al quirófano.

Es relevante mantener tranquilo al paciente en el cubículo de atención y durante su traslado al quirófano. Si se agita, inquieta o llora puede volverse a un escenario no controlado, se incrementan la generación de aerosoles y la posibilidad de contaminación. ${ }^{9}$ Flujograma 1

No debe descartarse la posibilidad de administrar medicamentos para sedación, teniendo en consi- 


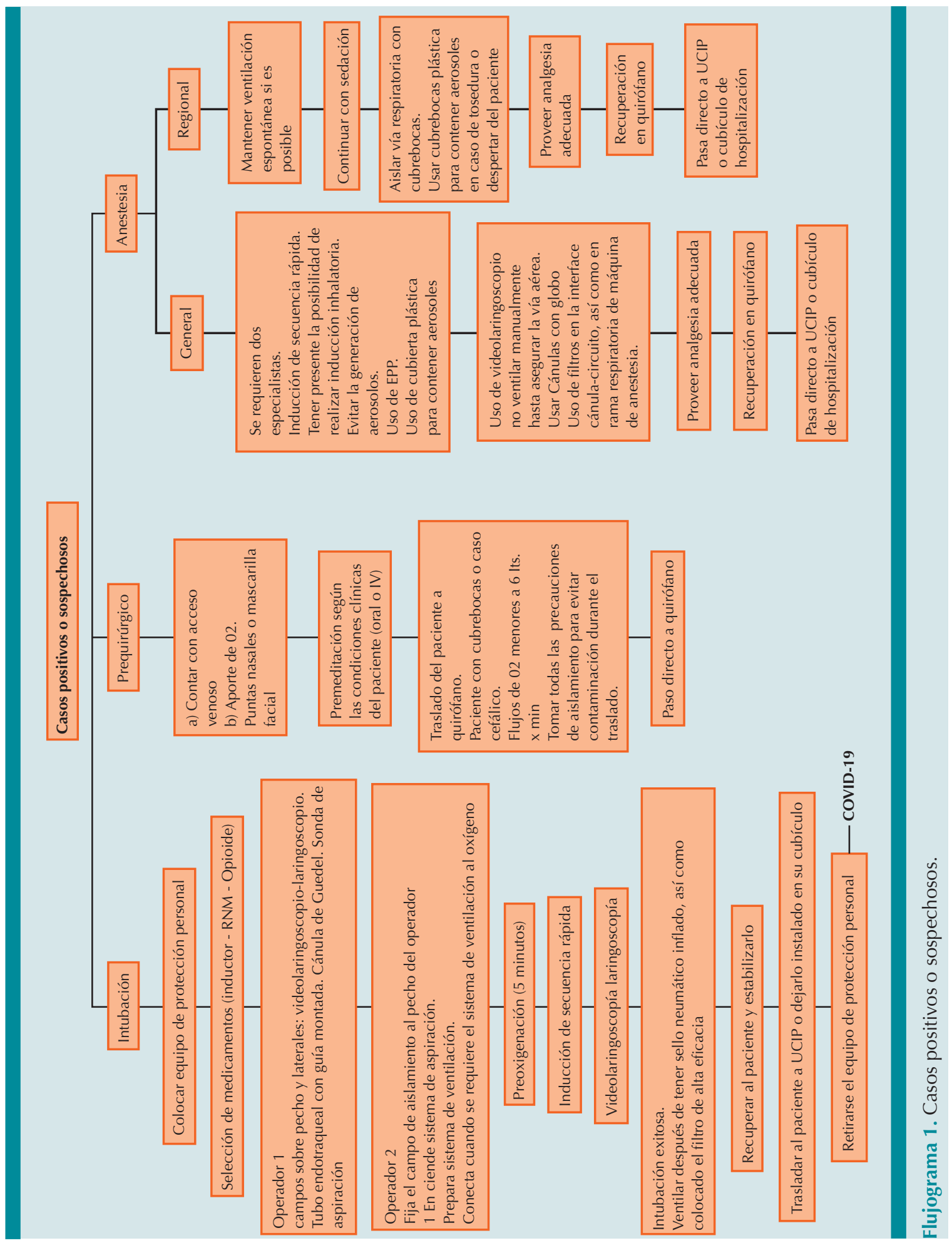


deración las condiciones clínicas del paciente, la baja tolerancia a la hipoxia, y los efectos de los medicamentos en la vía respiratoria.

Medicamentos. Son varias las opciones farmacológicas disponibles para sedar al paciente, pero no todas ofrecen el mismo margen de seguridad, por las implicaciones respiratorias que cada una genera.

Entre ellas está la dexmedetomidina, que es un alfa-2 selectivo que puede producir un adecuado grado de sedación sin depresión respiratoria, con margen de seguridad en la preservación de la función y de la vía aérea; igualmente, disminuye la incidencia de tos, y laringoespasmo a la hora de manipular la vía aérea. Tiene la gran ventaja de no desencadenar apnea, ni incrementar la secreción salival; su repercusión en el calibre de las vías aéreas es mínima; ${ }^{10,11}$ mayor con propofol, midazolam y halogenados. ${ }^{12,13,14}$

La dosis sugerida es de 1.5 -2 mcg por kg de peso intravenoso, a pasar lentamente, siempre con monitoreo continuo, tipo I como mínimo, y aporte de oxígeno. Es importante recordar que no es recomendable la administración en bolo intravenoso. Si se decide la administración por vía oral, se recomienda que la dosis no sobrepase los $2 \mathrm{mcg} / \mathrm{kg}$. La vía intranasal no se recomienda en estos casos. Se sugiere tener siempre al paciente monitorizado, por el efecto hemodinámico de la dexmedetomidina. ${ }^{15}$

Durante la vigilancia en el quirófano deberán seguirse los lineamientos de protección del personal, y la secuencia de intubación. Al igual que en las guías descritas para el paciente adulto, la técnica anestésica debe planearse acorde con el procedimiento a realizar y las condiciones del paciente. Recordar siempre disminuir al máximo la generación de aerosoles por el paciente o por los procedimientos que se le realicen. Al finalizar la cirugía, la recuperación debe tener lugar en el quirófano y después el paciente se traslada directamente a su cubículo de hospitalización, observando las mismas consideraciones de protección. Es importante seguir las estrategias de traslados de pacientes, incluidas el trazo de la ruta menos riesgosa y más rápida.

El contenido de este documento se limita a sugerencias tomadas de una revisión bibliográfica. Seguramente se omitieron algunos aspectos que el tiempo permitirá incluirlos. La intención fue proveer a los anestesiólogos un apoyo, a través de un panorama de principios y normas a seguir.

\section{REFERENCIAS}

1. Cook TM, et al. Consensus guidelines for managing the airway in children with COVID-19. Anaesthesia, 2020. https:// icmanaesthesiacovid-19.org/covid-19-paediatric-airwaymanagement-principles.

2. Center for Disease Control. Environmental Cleaning and Disinfection Recommendations: Interim Recommendations for US Households with Suspected/Confirmed Coronavirus Disease 2019. https://www.cdc.gov/coronavirus/2019ncov/community/home/cleaning- disinfection.html

3. Sinha R, et al. Paediatric Critical Care COVID-19 Guidance. 2020, de Paediatric Intensive Care Society. https://picsociety. uk/covid19/

4. Calvo C, et al. Recomendaciones sobre el manejo clínico de la infección por el «nuevo coronavirus» SARS-CoV2. Grupo de trabajo de la Asociación Española de Pediatría. An Pediatr (Barc). 2020. https://doi.org/10.1016/j.anpedi.2020.02.001

5. Vázquez $A$, et al. Bioseguridad y manejo de la vía aérea en el paciente crítico. Reporte especial COVID-19. Sociedad Científica Internacional EMIVA, 2020; 1-11.

6. Shannon L, et al. Simulation as a tool for assessing and evolving your current personal protective equipment: lessons learned during the coronavirus disease (COVID-19) pandemic. Can J Anesth. 2020. https://twitter.com/ShannonLockhart/status/ 1239032499994386433 (2020).

7. Montferrer N, et al. Guía de seguridad intraoperatoria y actuación frente a SARS-Cov-2. Hospital Universitari Vall d’Hebrón. Marzo 2020.

8. Silva J. Guía de actuación frente a SARS-Cov-2. Anestesiar.org. https://anestesiar.org/WP/uploads/2020/03/ Gu\%C3\%ADa-de-actuaci\%C3\%B3n-SARS-CoV-2-HospitaI12octubre.pdf 
9. Hervias $\mathrm{M}$, et al. Principales recomendaciones en el manejo anestésico del paciente pediátrico en el contexto actual de pandemia por Covid19. SEDAR. https://www.sedar. es/images/site/NOTICIAS/coronavirus/2020_Ped_Covid19_recomendaciones_final.pdf

10. Mahmoud M, et al. Effect of increasing depth of dexmedetomidine anesthesia on upper airway morphology in children. Pediatric Anesthesia. 2010; 20: 506-15. doi:10.1111/j.1460-9592.2010.03311.x

11. Subramanyam $R$, et al. Upper airway morphology in down syndrome patients under dexmedetomidine sedation. Rev Bras Anestesiol. 2016; 66 (4): 388-94. http://dx.doi. org/10.1016/j.bjane.2014.11.019

12. Yanmei B, et al. Efficacy of premedication with intranasal dexmedetomidine for removal of inhaled foreign bodies in children by flexible fiberoptic bronchoscopy: a ran- domized, double-blind, placebo-controlled clinical trial. BMC Anesthesiology; 2019;19. https://doi.org/10.1186/ s12871-019-0892-6

13. Lodenius A, et al. Upper airway collapsibility during dexmedetomidine and propofol sedation in healthy volunteers: A nonblinded randomized crossover study. Anesthesiology 2019; 131, 962-73.

14. Chen $\mathrm{H}$, et al. Intranasal dexmedetomidine is an effective sedative agent for electroencephalography in children. BMC Anesthesiology. 2020; 20: 61. https://doi. org/10.1186/s12871-020-00978-z

15. McAdams R, et al. Dexmedetomidine pharmacokinetics in neonates with hypoxic-ischemic encephalopathy receiving hypothermia. Anesthesiology Research and Practice. 2020. https://doi.org/10.1155/2020/2582965 